\title{
Design Study of a Miniaturized Multi-layered Antenna-in-package for 2.4 GHZ Wireless Communication
}

\author{
Jalal Naghar ${ }^{1}$, Otman Aghzout ${ }^{2}$, Azzeddin Naghar ${ }^{3}$ \\ ${ }^{1}$ Dept. of Physics, Faculty of Science, Abdelmalek Essaadi University, Tetouan, Morocco \\ ${ }^{3}$ Electronics \& Microwave Group, Telecommunication Department, ENSA, Tetouan, Morocco \\ ${ }^{2}$ Dept. of Signal theory and Communications, University of Vigo, Vigo, Spain
}

\begin{tabular}{l} 
Article Info \\
\hline Article history: \\
Received Mar 3, 2018 \\
Revised Jul 28, 2018 \\
Accepted Aug 5, 2018 \\
\hline
\end{tabular}

Keyword:

$2.4 \mathrm{GHz}$ band

Compact size

Miniaturization techniques

Multi-layer

Multilayered antenna

\begin{abstract}
This paper proposes a novel miniaturization technique to enhance the radiation properties of small multi-layer patch antenna used in packaged circuits. The multilayered antenna design is composed of three layers with different shapes. An enhancement on the radiation properties has been obtained by optimizing the geometry of the radiated element and the parasitic conductor of the middle layer. The whole design has been implemented on the FR4 substrate with dielectric constant of 4.4, thickness of $1.6 \mathrm{~mm}$ and Copper thickness of $5 \mu \mathrm{m}$. The first layer is a driven element while second and the third layer are parasitic patch elements. The optimized multilayer antenna has a very small size of $12 \times 6 \times 5 \mathrm{~mm}^{3}$. Considering the small size of the antenna, a detailed study of the parameter affecting the radiation has been considered to force the antenna to operate at $2.4 \mathrm{GHz}$ band. Miniaturization techniques based on the current distribution have been also taken into account to shift down the resonant frequency and reduces more and more the antenna size at the designed operating frequency. The miniaturized antenna maintains performant radiation characteristics in terms of reflexion coefficient, bandwidth and directivity. All developed antennas are simulated using the commercial Electromagnetic CST Microwave Studio software. Achieved results demonstrate a good performance with low cost and compact size
\end{abstract}

Copyright () 2018 Institute of Advanced Engineering and Science. All rights reserved.

\section{Corresponding Author:}

Jalal Naghar,

Dept. of Physics, Electronic and Microwave Group,

Faculty of Science,

Abdelmalek Essaadi University,

Tetouan, Morocco.

Email: jalalnaghar@gmail.com

\section{INTRODUCTION}

From the progressing of communication systems, antenna design has been always the prime important to take up this challenge. Microstrip patch antennas are very attractive candidates for use in modern wireless communication systems due to their moderate performance and ease of integration with microwave devices and wireless engineers [1], [2]. The microstrip antenna is more and more frequently in modern communication systems, especially in wireless system for local area network [3]. Therefore, it is challenging to design and miniature microstrip antennas to have better radiating properties with a compact size. In the literature concerning the miniaturization of the size of patch antennas, authors use several techniques. Some of them consist on insertion of shorting posts and pin [4], short circuit [5] and cutting slots in radiating patch [6], [7]. The optimization is obtained also by partially filled high permittivity substrate[8], using fractal microstrip patch configuration [9] and increasing the electrical length of the antenna by optimizing the shape [10] and ching periodical slow wave structures on the ground plane [11]. To reduce the 
antenna dimensions mostly shorted patches and PIFAs are used [12]-[14]. In 2010 presented a planar electrically small rectangular microstrip patch antenna loaded with metamaterial square split ring resonator (SRR) [15] . In this paper multilayered technology is used for reduce the size of the Microstrip patch antenna. This is very simple, efficient, very useful for antenna design engineers to design small antenna for wireless communication.

A small multilayer antenna mounted on three layers is proposed in this contribution, to operate in the Industrial, Scientific and Medical (ISM) frequency band at $2.45 \mathrm{GHz}$. The antenna has three layers separated by air gapping with feed line in the first, a slotted radiating patch in the second and a square parasitic patch in the third layer. The FR4 substrates with a Cooper thickness of $50 \mu \mathrm{m}$ are combined together to obtain the best results and design for the proposed antenna. An optimization study of size and performance based on different shapes for the two parasitic patch in the second and third layers. The resulting antenna configurations are presented and compared, between different structures presented in this work. The total size, center frequency and bandwidth of each case are studied in detail to achieve the optimal design. The antenna proposed with circular aperture and circular parasitic element is miniaturized for obtain low resonance frequencies based on the effect of length of the feed line and radius of aperture.

The CST MWs electromagnetic simulator is used to perform the antenna characteristics. These performances demonstrate that the proposed antenna is good candidate wireless communication systems operating at $2.4 \mathrm{GHz}$. This paper is divided into four sections: the first section is devoted to give the geometry design of the multi-layers patch antenna and a preface of the important parameters in different layers designs, for top layer, middle layer and bottom layer. The result obtained and the design consideration are discussed in this section. Second section present the geometry study of this antenna on the antenna responses, based on effect different shapes of the radiated elements such as the aperture in the second layer and parasitic patch in the third layer. The shapes study of the parasitic patch are circular, fractal and square combined by circular, fractal and square forms of the aperture in the middle layers. A comparison between all combinations are presented and discussed in this section. Third section presents an antenna geometry of the case having a good radiation properties. Other type of antenna miniaturization is proposed in this section. Finally, a brief conclusion is presented in the fourth section.

\section{ANTENNA DESIGN}

In this section, it is explained the specification of the geometry design of the multi-layers patch antenna proposed, we provide a description for different layers designs, and a preface of the radiated shapes using in this study.

\subsection{Multilayers patch antenna for $2.4 \mathrm{GHz}$}

Our objective in this paper is to design a novel very small antenna based on wireless application systems developed previously [10], [11]. The proposed multi-layer structure antenna is composed by multilayers substrates and multi-conductor. A long detailed study on the parameter antenna it was taken into account to miniaturize the dimensions of the antenna, precisely the effect of the slotted radiating patch and square parasitic element. After a long procedure design, an exploded view of this antenna is can be shown in Figure 1.

As a first step, the antenna is implemented on FR4 substrate having a relative permittivity of 4.4 and thickness of $1.6 \mathrm{~mm}$. The ground plane dimensions are $\mathrm{W}_{-} \mathrm{g} \times \mathrm{L} \_\mathrm{g}=6 \times 6 \mathrm{~mm}^{2}$. The microstrip patch is feed by microstrip of width $\mathrm{W}_{\mathrm{f}}=1 \mathrm{~mm}$ printed on bottom dielectric layer. The feed line and partial ground plan are chosen to be copper with thickness of $0.05 \mathrm{~mm}$, which are separated by the FR4 substrate of $1.6 \mathrm{~mm}$. The antenna presented is based in multi layers techniques on the FR4 substrate. Introducing slots in radiating patch with different geometry allow big performance and important improvement in size dimensions. Figure 1 presents a detailed initial design of $2.4 \mathrm{GHz}$ proposed antenna-in-Package. The rest of parameters of the designed antenna are: $\mathrm{L}_{\mathrm{f}}=5.1 \mathrm{~mm}, \mathrm{~W}_{\text {slot }}=\mathrm{L}_{\text {slot }}=4.8 \mathrm{~mm}, \mathrm{~L}_{\mathrm{p}}=5.5 \mathrm{~mm}, \mathrm{~W}_{\mathrm{p}}=5.5 \mathrm{~mm}$ and tree layers of antenna having fixed values of $\mathrm{L}_{\mathrm{s}} \times \mathrm{W}_{\mathrm{s}} \times \mathrm{H}_{\mathrm{s}}=6 \times 6 \times 1.6 \mathrm{~mm}^{3}$.

The antenna consists of three layers with slotted radiating patch and square parasitic located on the middle and top layer respectively. The parasitic patch is located at the upper side of the top dielectric layer in order to provide a maximum radiation; Aperture in the middle patch is used to radiate the parasitic element.

We present the antenna radiation behavior after studying and optimizing all critical parameters, Figure 2 shows the simulated return loss performance of the developed antenna.

It can be observed that the proposed antenna achieves a bandwidth of 1.4 to $3.5 \mathrm{GHz}$. The plotted result indicates that, at $-48 \mathrm{~dB}$ return loss, the compact antenna operate perfectly at $2.4 \mathrm{GHz}$. Consequently, the antenna presented based in multilayer substrates and dimension parameters techniques to reduce the size, provides good radiations properties. 


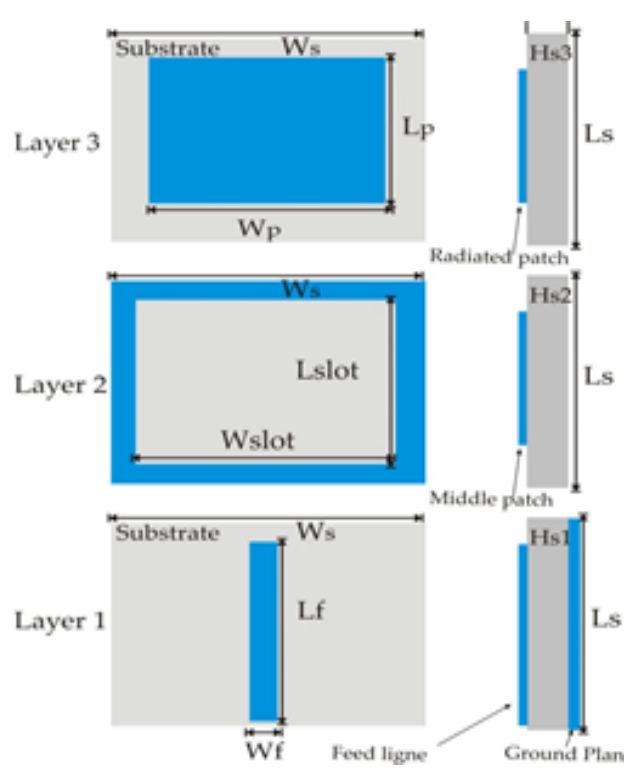

(a)

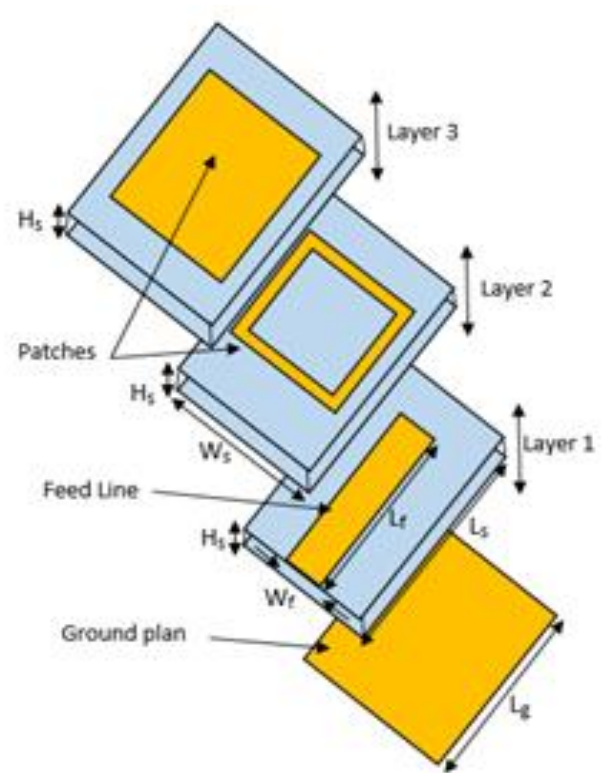

(b)

Figure 1. Geometry of the proposed very small antenna using FR4 (a) by layers, (b) : 3D

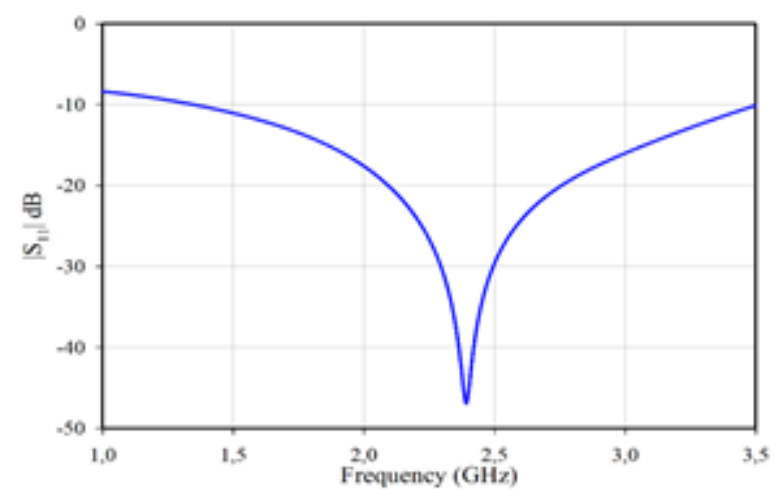

Figure 2. Return Loss of the proposed antenna on FR4 material

By comparing the structure developed in Figure 1, With the conventional single layer microstrip antenna operating in the same resonance frequency of $2.4 \mathrm{GHz}$, we conclude that $80 \%$ reduction in surface size antenna enhancement is obtained offering good radiation properties with improvement of bandwidth with a return loss of about $-50 \mathrm{~dB}$.

\subsection{Design consideration}

The geometry proposed in this work is very small, for this raison is not possible to feeding the antenna by coaxial SMA coaxial probe connecter. In order to fabricate this antenna we propose to introduce the simple technique; this is expanding the feed line on $6 \mathrm{~mm}$ to be exciting the antenna. The new geometry proposed is presented in Figure 3. 


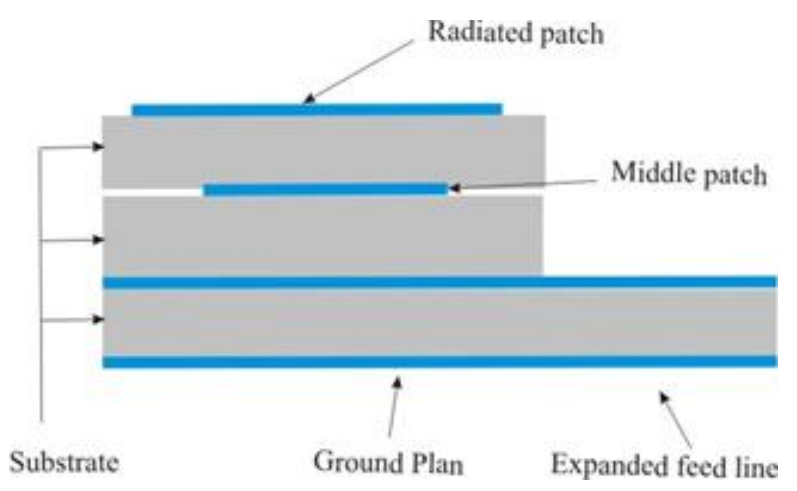

Figure 3. Design consideration of the proposed antenna

\subsection{Shapes effect of the radiated and parasitic patchs of the proposed antenna}

In this section, we present the shapes of the radiated elements structure and parasitic patch used in this study, we propose to study all possible combinations of different shapes on the radiating patch presented in Figure 4 (square, circular, and fractal) and different forms of aperture in Middle patch (square aperture, circular aperture and fractal aperture) illustrated in Figure 5, All scenario models are presented in Table 1.

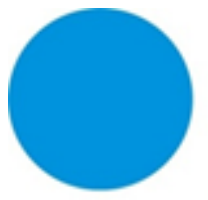

(a)

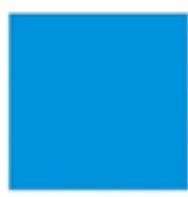

(b)

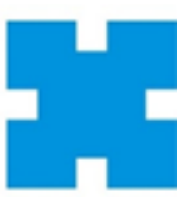

(c)

Figure 4. Proposed parasitic patch shapes

(a) circular, (b) squar, (c) fractal

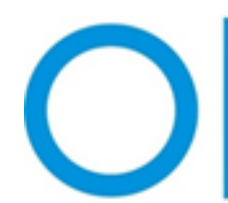

(c)

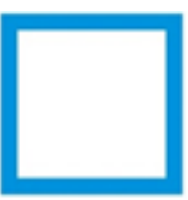

(f)

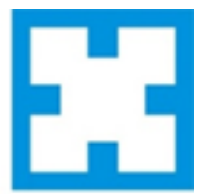

(g)

Figure 5. Proposed aperture shapes (middle patch) (a) circular, (b) squar, (c) fractal

Table 1. Different Combinations Scenarios for the Multilayer Antenna under Study

\begin{tabular}{cccc}
\hline Parasitic & \multicolumn{3}{c}{ Aperture of the middle patch } \\
patch & Square & Circular & Fractal \\
\hline Square & Case 1 & Case 4 & Case 7 \\
Circular & Case 2 & Case 5 & Case 8 \\
Fractal & Case 3 & Case 6 & Case 9 \\
\hline
\end{tabular}

In the next step, a detail study of the different radiated shapes combinations can demonstrate clearly, the effect of these shapes on the desired response. As can be shown below, the form of the aperture middle patch has an important effect on the current distribution of the antenna presented.

\section{RESULTS AND ANALYSIS}

In this section, we present the the effect of radiated elements structure (radiated and parasitic patch) on the antenna responses. The aim of this study is to demonstrate that, by implementing radiated elements with different shapes it is possible to obtain the antenna responses with higher improvements in term of bandwidth, return loss and reductions in volume size. 


\subsection{Antenna with square aperture}

In this section, we present the effect study of parasitic patch implemented in third layer of antenna proposed on the antenna responses. For this purpose, the aperture form in the second layer has a square form (Csae1, Case2, and Case3 in Tab1) and the results are plotted in Figure 6.

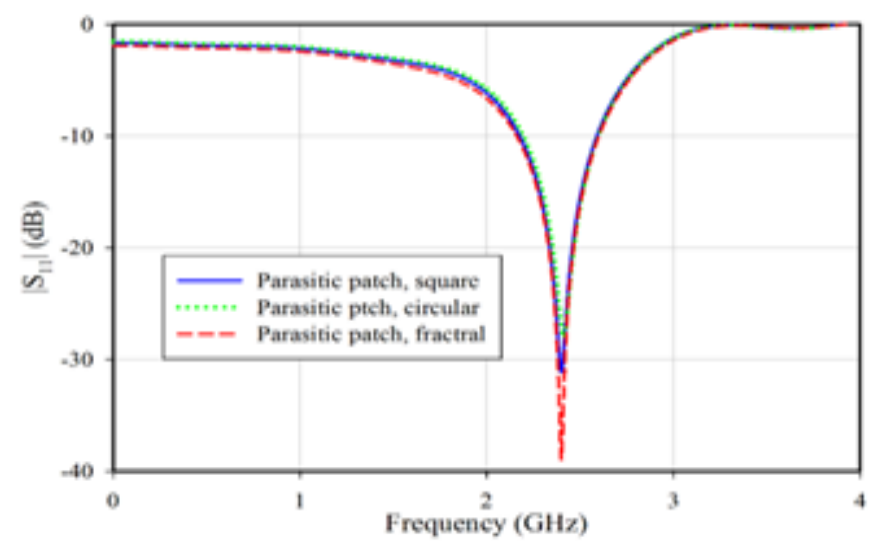

Figure 6. Return Loss of the proposed antenna with square aperture for different radiated element structures

\subsection{Antenna with circular aperture}

As mentioned previously, our principal purpose is focused on the study of the effect of the aperture in the middle patch on radiation properties of this antenna. In this section, we will present the case 3 , Case 4 and case 5 from Table 1.

Figure 7 shows the simulated Return loss of the proposed antenna with circular aperture using different form of the parasitic element in the top layer. After a detailed study, we consider that, the circular aperture have a big effect on the resonance frequency and bandwidth of the antenna, it is clear that the implementation of circular radiated patch with circular aperture (Case 5) allow big performance and important improvement in bandwidth. Therefore, the radiation of this antenna is more important with the fractal parasitic patch (Case 6).

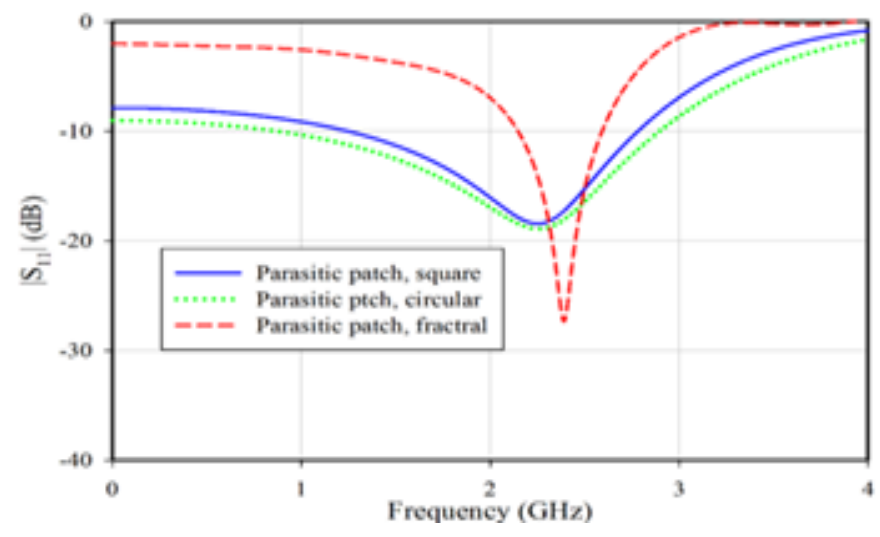

Figure 7. Return Loss of the proposed antenna with circular aperture for different radiated element structures

\subsection{Antenna with fractal aperture}

The effect of radiated patch geometry on the antenna radiations properties is presented in this section with the Fractal aperture in the middle layer. The return loss of the proposed multi-layer antenna (Case 7, Case 8 and Case 9) is presented in Figure 8.

We can observe that, the geometry of the radiated element affects the radiation and resonance frequency of the antenna. Therefore for the circular parasitic element have an important effect on the antenna response with return loss of $-40 \mathrm{~dB}$. 


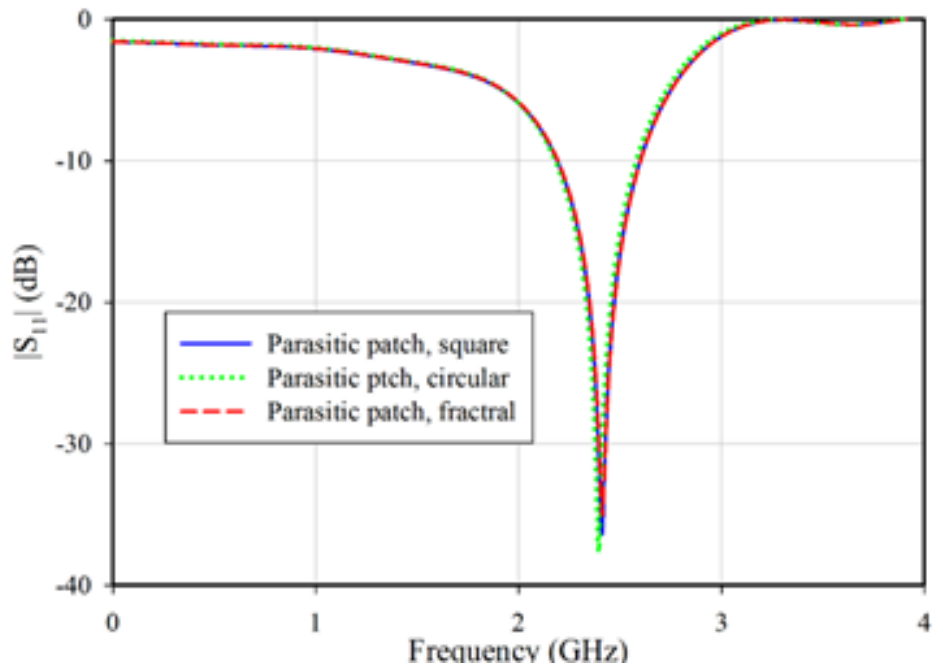

Figure 8. Return Loss of the proposed antenna with fractal aperture for different radiated element structures

\subsection{Results and coparisons}

The aim of this study is demonstrate that the form of the shape radiated in the patch has a big effect in the radiation properties on this antenna on frequency resonance of $2.4 \mathrm{GHz}$. For this raison different cases presented in Table 1 are presented in the work. The good result obtained in the different cases for this study is illustrated in Table 2. The return loss of the antenna proposed for the optimized cases is show in Figure 9.

Table 2. Optimized Combinations Scenarios for the Multilayer Antenna under Study

\begin{tabular}{cccccc}
\hline Design & frequency & Bandwidth & Directivity & VSWR & Radiation (dB) \\
\hline Case 3 & 2.4 & $435.2 \mathrm{MHz}$ & 2.45 & 1.05 & $-44 \mathrm{~dB}$ \\
Case 5 & 2.4 & $1880 \mathrm{MHz}$ & 3.31 & 1.3 & $-21 \mathrm{~dB}$ \\
Case 7 & 2.4 & $400 \mathrm{MHz}$ & 2.53 & 1.02 & $-45 \mathrm{~dB}$ \\
\hline
\end{tabular}

The case 5 present a good performance and important improvement in bandwidth. Therefore, the radiation of this antenna is more important in case 3 and case 7 . We conclude that the antenna in package with circular radiation element and circular aperture (Case 5) is the best combination for obtained good radiation properties. The fabrication results is the principal factor for determinate the optimized structure.

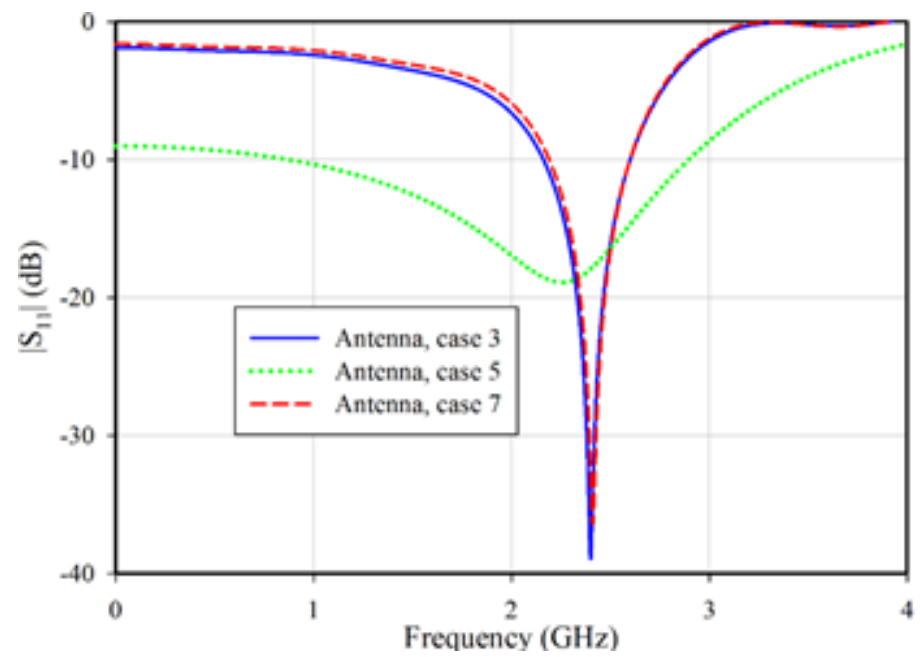

Figure 9. Return Loss of the proposed antenna of the antenna proposed for the optimized cases 


\subsection{Antenna miniaturization}

The geometry of the optimized antenna structure is shown in Figure 10. The circular parasite patch and circular aperture have an important effect for miniaturized of this antenna this structure have a good performance in a bandwidth in comparison with others structure in Table 2. The most important parameter in view of this structure is the radius of the circular aperture, he affect the radiation properties of this antenna.

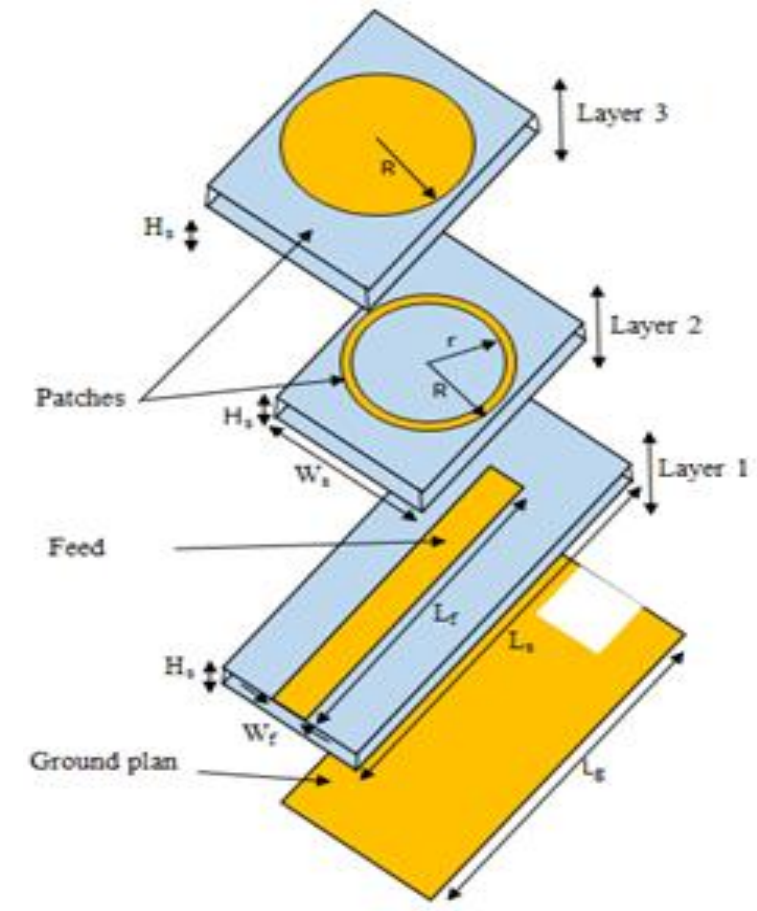

Figure 10. Geometry in detail of the optimized antenna

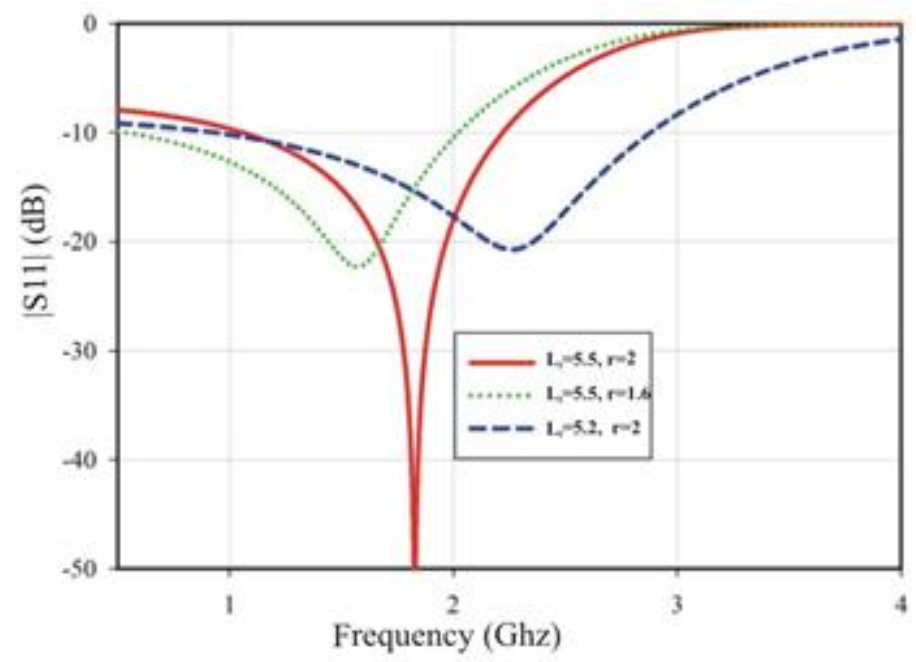

Figure 11. Return Loss of the proposed antenna with for different radius of the aperture

It should be noted that, a study of the proposed antenna with different shapes of the previous paragraph is necessary to obtain the best responses in the same resonance frequency. A study of the critical parameters effect of the optimized antenn is introduced to improve more and more the antenna performance and miniature this optimized structure proposed in this work. 
We conclude in this study that the radius of the aperture and length of feed line are the two critical parameters to determine the response of this antenna. Many scenarios are tried to choice the optimum radius of the circular aperture for the antenna optimized to give maximum miniaturization result of the antenna, Figure 11 illustrates the antenna response for different values combination between radius $r$ of the circular aperture and length of feed line Lf.

As shown in the figure, it is clear that the $\mathrm{Lf}$ and $\mathrm{r}$ radius are responsible to shifting the frequency band. The variation values of this two parameter include the low frequency, this is another type of miniaturization of this antenna.

\section{CONCLUSION}

In this paper a very small antenna for packaging systems at $2.4 \mathrm{GHz}$ frequency band technologies is presented. Multi-layer substrate with FR4 has been introduced. Study based on the different shapes in the parasitic patch and aperture element is implemented to the response of the proposed antenna. All combination study are optimized for obtained frequency radiated of $2.4 \mathrm{GHz}$. The antenna study having a circular shapes in the radiated patch and parasitic patch have the best radiation properties in comparing with others structures study in this word. Because the radius of the aperture and length of feed line are the two principal factor to determine the antenna response, this antenna is miniaturized for obtained the low resonance frequency. This technique discussed here may be extended to other geometries like triangular, diagonal or fractal patches and may find potential applications.

\section{ACKNOWLEDGEMENTS}

Research supported by Electronics \& Microwave Group, Telecommunication Department of National School of Applied Sciences of Tetouan (Tetuan, Morocco).

\section{REFERENCES}

[1] Rohit K. Raj, Monoj Joseph, C.K. Anandan, K. Vasudevan, P. Mohanan, “A New Compact Microstrip-Fed DualBand Coplaner Antenna for WLAN Applications”, IEEE Trans. Antennas Propag., vol. 54, no. 12, pp. 3755-3762, 2006.

[2] Zhijun Zhang, Magdy F. Iskander, Jean-Christophe Langer and Jim Mathews, "Dual-Band WLAN Dipole Antenna Using an Internal Matching Circuit”, IEEE Trans. Antennas and Propag., vol. 53, no. 5, pp. 1813-1818, 2005.

[3] K.F. Tong, K.M. Luk, K.F. Lee and S.M. Shum, "Analysis of a broadband U-slot Microstrip Antenna", 10th International Conference on Antenna and Propagation, no. 436, pp. 14-17, 1997.

[4] K.-L. Wong, Compact and Broadband Microstrip Antennas, John Wiley \& Sons, New York, NY, USA, pp. 1-7, 2002.

[5] S. Pinhas and S. Shtrikman, "Comparison between Computed and Measured Bandwidth of Quarter-wave Microstrip Radiators", IEEE Transactions on Antennas and Propagation, vol. 36, no. 11, pp. 1615-1616, 1988.

[6] K. L. Wong and K. P. Yang, "Small dual Frequency Microstrip Antenna with a Cross Slot", Electronics Letters, vol. 33, pp. 1916-1917, 1997.

[7] Kuldeep Kumar Parashar, "Design and Analysis of ISlotted Rectangular Microstrip Patch Antenna for Wireless Application", International Journal of Electrical and Computer Engineering, vol. 4, no. 1, pp. 31-36, 2014.

[8] Y. D. Lin and T. Itoh, "Frequency-scanning Antenna using the Crosstieoverlay slow-wave Structures as Transmission Line", IEEE Trans Antennas Propag., vol. 39, pp. 377-380, 1991.

[9] Satyandra Singh Lodhi, P.K. Singhal and V.V. Thakare, "Design and Analysis of Tripple Band Koch Fractal Yagi Uda Antenna", International Journal of Electrical and Computer Engineering, vol. 3, no. 4, pp. 456-460, 2013.

[10] N. Herscovici and M. F. Osorio et al., "Miniaturization of Rectangular Microstrip Patches using Genetic Algorithms", IEEE Antennas Wireless Propag.Lett., vol. 1, pp. 94-97, 2002.

[11] B. Lee and F. J. Harackiewicz, "Miniature Microstripantennawith a Partially Filled high - Permittivity Substrate", IEEE Transactions on Antennas and Propagation, vol. 50, no. 8, pp. 1160-1162, 2002.

[12] H. K. Kan, R. B. Waterhouse, "Small Printed-wing Antenna Suitable for Wireless Handset Terminals", Microwave and Optical Technology Letters, vol. 30, no. 4, pp. 226-229, 2001.

[13] Shackelford, A. K., K. F. Lee, and K. M. Luk, "Design of small-size wide-bandwidth Microstrip-patch Antennas", IEEE Anten. and Propa. Mag., vol. 45, no. 1, pp. 75-83, Feb. 2003.

[14] R. Chair, K. M. Luk, K. F. Lee, "Miniature shorted Dualpatch Antenna”, IEE Proceedings - Microwaves, Antennas and Propagation, vol. 147, no. 4, pp. 273-276, 2000.

[15] J. G. Joshi, S. S. Pattnaik, S. Devi and M. R. Lohokare, "Electrically Small Patch Antenna Loaded with Metamaterial”, IETE Journal of Research, vol. 56, no. 6, pp. 373-379, 2010. 


\section{BIOGRAPHIES OF AUTHORS}
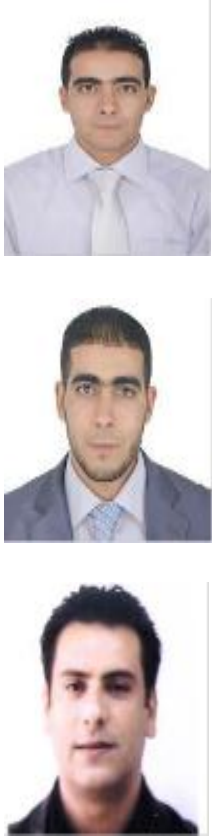

He was born in Tetouan, Morroco. He received the Engineer Degree in telecommunication engineering at the National School of Applied Sciences from Abdelmalek Essaadi University, Tetouan, Morocco 2011. He is currently working toward the Ph.D. degree in Telecommunications Engineering with the electronic and microwave group at Abdelmalek Essaadi University. His research interests include antennas and systems in package.

He was born in Tetouan, Morroco. He received the Engineer Degree in telecommunication engineering at the National School of Applied Sciences from Abdelmalek Essaadi University, Tetouan, Morocco 2011. He is currently working toward the Ph.D. degree in Telecommunications Engineering with the electronic and microwave group at Abdelmalek Essaadi University. His research interests include antenna design and RF filters.

He was born in Tétouan, Morocco. He received the Electronics degree from Abdelmalek Essaadi University, Tétouan, Morocco, in 1995, and $\mathrm{M}$. degree and the Ph.D. degree in Telecommunications Engineering at the High School of Telecommunications Engineering (ETSITGC) of Canary University, in 2002. Since 2009 He joined the Telecommunication Departmentt as an associate Professor of Telecommunications Engineering at the National School of applied Sciences, UAE, Tetouan, Morocco. Currently he is interested on printed microwave passive and active circuits, Filters and antenna designs. 